\title{
Nefrectomia total unilateral em cachorro-do-mato (Cerdocyon thous)
}

\author{
Total Unilateral Nephrectomy in a Crab-eating fox (Cerdocyon thous) \\ Ronaldo José Piccoli', Dhyego Thomazoni², Juliana Teixeira Druziani², \\ Márcio Hamamura ${ }^{3} \&$ Anderson Luiz de Carvalho ${ }^{4}$
}

\begin{abstract}
Background: The loss of natural habitats associated with the development of humanity resulted in the need for longer trips and greater interaction with highways by some animal species. Vehicle collision with animals is one of the main factors of pressure over wild animal populations in Brazil; veterinary surgery can play an important role for the recovery of these patients. The lesions commonly seen in animals hit by vehicles may comprise skin lesions, central nervous system injuries, intrathoracic and intra-abdominal complications, among which renal lesions are included. The aim of this work is to report the use of a total nephrectomy technique in a crab-eating fox.

Case: A female, adult, 5-kg crab-eating fox (Cerdocyon thous Linnaeus, 1766) was received for emergency care at the Veterinary Hospital of Universidade Federal do Paraná at Palotina. The patient had been struck by a vehicle at a local highway, was unconscious, and exhibited signs of blood loss such as hypothermia, hypotension, and pale mucous membranes. As the clinical evaluation was performed, the patient was stabilized with pain management, correction of hypovolemia and hypothermia, and oxygen therapy. Blood samples were taken in case of the need for preoperative tests. Subsequently, the animal was submitted to the diagnostic imaging section to investigate possible injuries. No chemical restraint was necessary for the procedures. Radiography examination revealed loss of intra-abdominal contrast, which was confirmed to be free fluid on ultrasound examination. Immediately after imaging examination, the animal was taken to the surgical room for exploratory laparotomy. The surgical incision, which was performed over the linea alba, extended from the xiphoid process to the pubis. During surgery, free fluid was confirmed to be diffuse bloody fluid, which was observed in the abdomen; there was absence of lesions in all organs except the right kidney, which revealed clots and lacerations to the capsule, cortex, and medulla, resulting in poor viability of the organ. The condition required removal of the organ, which was performed with the conventional technique described for domestic animals. During the nephrectomy surgical procedure, the patient exhibited severe hypotension with the need of compensatory measures such as intravenous administration of crystalloid and colloid solutions, and vasoactive drugs, which were maintained during the period of surgery. Postoperative care included administration of analgesics and anti-inflammatory drugs for three days, and antibiotics for seven days. Hematological and renal profiles as well as ultrasound examinations were used for monitoring of clinical evolution. The patient was kept in isolation to avoid behavioral stress during hospital stay.

Discussion: In this case, the importance of ultrasound diagnosis and viability of the nephrectomy technique were established for the specimen, which was returned to its natural habitat eighteen days after the vehicle collision, after authorization from the local environmental agency. Immediate care of a wild animal that suffers trauma is an important factor to increase success rate for recovery of fauna threatened by vehicle collisions. Early diagnosis associated with the employment of surgical techniques commonly used for domestic animals allow wild-animal clinicians and surgeons to improve viability of threatened populations.
\end{abstract}

Keywords: crab-eating fox, wild animal, nephrectomy.

Descritores: cachorro-do-mato, animais silvestres, nefrectomia. 


\section{INTRODUÇÃO}

O atropelamento de fauna silvestre é uma das principais causas do declínio de populações de animais silvestres de vida livre [2], e considera-se que as taxas de ocorrência de atropelamentos sejam subestimadas, pois os animais que não morrem no momento da colisão deslocam-se para a vegetação adjacente sem serem contabilizados [25]. O cachorro-do-mato (Cerdocyon thous) apresenta ocorrência em vários países além do Brasil, e possui hábitos crepusculares e noturnos, com dieta onívora e generalista [1,21,22]. Apesar dessa espécie não estar listada como ameaçada de extinção, diversas populações acabam sofrendo importantes impactos por meio de atropelamentos nas rodovias do país [14,21,22].

Em cachorros-domésticos, lesões em diversos sistemas do organismo são descritas em $30 \%$ dos animais traumatizados, e comumente os casos de óbito são decorrentes de lesões intratorácicas, intra-abdominais e no sistema nervoso central [7]. Em humanos, dentre as causas de lesões renais incluem-se os traumas fechados, como atropelamentos [5,8,12], e o mesmo é observado para animais domésticos [6]. O procedimento de nefrectomia é indicado para afecções unilaterais, como: hidronefrose, neoplasias renais, destruição traumática de significativa parte do parênquima renal, entre outros [24].

O presente trabalho objetiva relatar o emprego da técnica de nefrectomia total unilateral em um espécime de Cerdocyon thous e contribuir na complementação de estratégicas terapêuticas para animais silvestres.

\section{CASO}

Uma fêmea adulta de Cerdocyon thous (cachorro-do-mato) atropelada nas coordenadas $24^{\circ} 16^{\prime} 38.51^{\prime \prime}$ Sul e 5246'22.83" Oeste, da rodovia PR-364, que liga as cidades de Palotina e Assis Chateaubriand no Paraná, foi recebida para atendimento emergencial. A paciente, com peso de $5 \mathrm{~kg}$, apresentava mucosas pálidas, frequência cardíaca de 120 batimentos por minuto, temperatura retal de $35,3^{\circ} \mathrm{C}$, pulso fraco, estupor, hematúria e rigidez e dor a palpação abdominal. Observou-se também soluções de continuidade em pele da extremidade distal do membro pélvico direito e cabeça (região de osso frontal) sugestivos de atrito com solo ou pista de rolamento.

Concomitante a avaliação clínica, procedeu-se o acesso venoso e proveu-se analgesia com cloridrato de tramadol (Tramadon ${ }^{1}, 4 \mathrm{mg} / \mathrm{kg}$, i.m.), correção da hipotermia e realizou-se coleta de sangue para exame de hemograma e bioquímica sérica. $\mathrm{O}$ animal foi conduzido ao setor de diagnóstico por imagem para realização dos exames de radiografia (projeções radiográficas ortogonais de tórax, abdômen, crânio e membros pélvicos/torácicos) e ultrassonografia (FAST-focused assessment with sonografy for trauma). Por apresentar estado de estupor não foi necessária nenhuma contenção química do animal, sendo o mesmo contido fisicamente para ambos os exames.

A análise do estudo radiográfico não indicou lesões em tecido ósseo, tampouco alterações em parênquima pulmonar ou em cúpula diafragmática, todavia, observou-se perda do contraste intra-abdominal, sugestivo de efusão peritoneal. No exame ultrassonográfico " $F A S T$ ", foi evidenciado presença de conteúdo anecogênico (líquido livre) em todos os recessos avaliados (Figura 1), contudo ao ser executada a abdominocentese não foi possível a recuperação de líquido. Tendo em vista as alterações apresentadas nos exames de imagem, o paciente foi encaminhado ao centro cirúrgico para realização de laparotomia exploratória. A indução anestésica foi realizada com propofol (Propovan ${ }^{\circledR}{ }^{1}, 4 \mathrm{mg} / \mathrm{kg}$, i.v.) e o paciente foi mantido com isoflurano (Isoforine ${ }^{\circledR}{ }^{1}$, inalatório) em vaporizador universal em quantidade suficiente para manutenção de plano adequado para cirurgia abdominal, caracterizado por rotação de globo ocular e reflexo palpebral e tônus muscular ausentes. Ao paciente foi permitido ventilação espontânea e esta era assistida quando a capnografia ultrapassava $50 \mathrm{mmHg}$. As variáveis monitoradas foram: eletrocardiograma, pressão

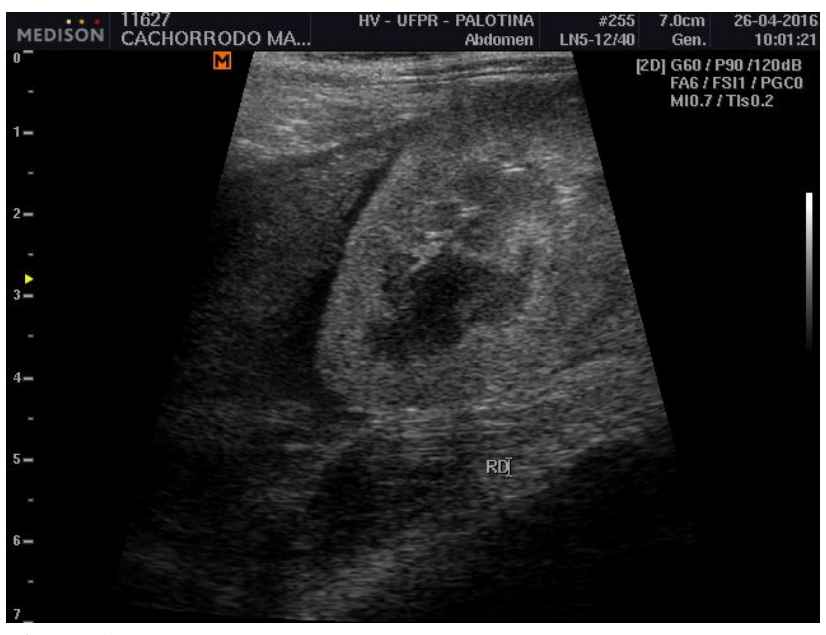

Figura 1. Imagem ultrassonográfica de rim direito (corte longitudinal recesso hepatorrenal). Observa-se moderada quantidade de coleção líquida anecogênica (melhor evidenciado em polo caudal renal), sugestivo de líquido livre abdominal. Adjacente, observa-se aumento de ecogenicidade mesentérica - peritonite focal. 
arterial por oscilometria, saturação de oxigênio e capnografia, sendo estes verificados a cada 5 min por meio de monitor multiparamétrico (Digicare ${ }^{\circledR}{ }^{2}$ LifeWindowLW9xVet). A fluidoterapia inicial foi estabelecida na taxa de infusão de $10 \mathrm{~mL} / \mathrm{kg} / \mathrm{h}$ com solução de $\mathrm{NaCl}$ 0,9\% (Cloreto de Sódio 0,9\% ®3).

A incisão cirúrgica em linha média se estendeu desde processo xifóide até a região púbica. Na inspeção da cavidade abdominal observou-se conteúdo sanguinolento difuso, que foi removido com o auxílio de um aspirador cirúrgico, com posterior limpeza da cavidade com solução de $\mathrm{NaCl}$ 0,9\% (Cloreto de Sódio 0,9\% ®3). Concomitante a remoção do líquido cavitário procedeu-se a inspeção minuciosa de cada órgão, buscando as possíveis origens do sangramento. $\mathrm{Na}$ inspeção renal foi constatada a presença de coágulos e lacerações de cápsula, córtex e medula do rim direito (Figura 2), e ausência de comprometimento do hilo renal. Frente ao significativo comprometimento do parênquima renal, optou-se pela remoção do órgão com a técnica de nefrectomia descrita por Rosin [24], onde após a mobilização do órgão a ser removido dissecou-se o hilo renal para a exposição do ureter, artéria e veia renal. Na sequência dissecou-se o ureter para a ligadura dessa estrutura o mais próximo possível da vesícula urinária, e posteriormente realizou-se a secção do ureter entre as ligaduras. Após esta etapa elevou-se o rim e dissecou-se a artéria e veia renal, ligando-as independentemente, realizou-se também a transfixação destes vasos, objetivando impedir sua retração a partir da ligadura. Findada a retirada do órgão, foram reposicionados os intestinos e o omento maior na posição anatômica, e realizada a síntese da cavidade abdominal de maneira usual.

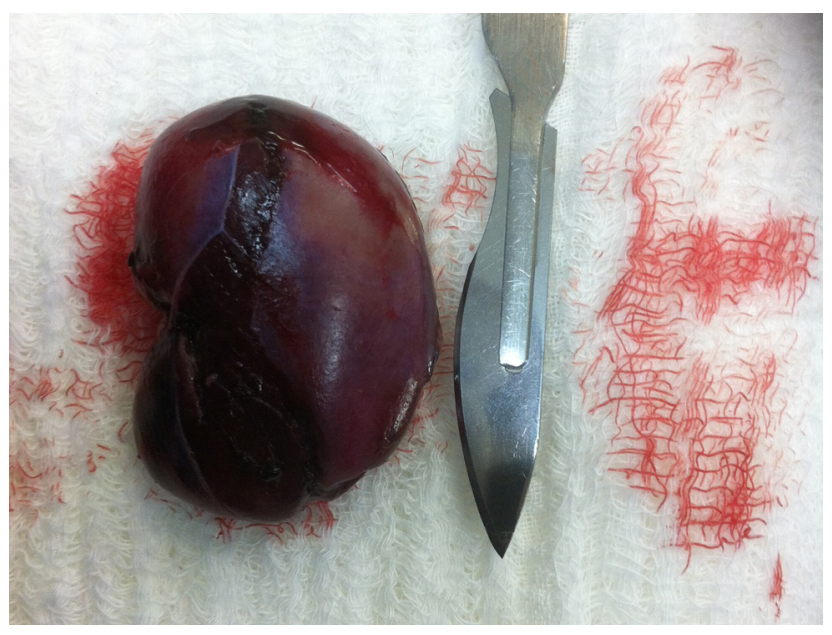

Figura 2. Visualização da lesão em rim direito do espécime de Cerdocyon thous após o procedimento de nefrectomia.
No decorrer do procedimento anestésico o paciente apresentou pressão arterial sistólica de 60 mmHg caracterizando quadro de hipotensão, que foi tratado com bolus de $\mathrm{NaCl}$ 0,9\% (Cloreto de Sódio $0,9 \% \AA^{3}, 10 \mathrm{~mL} / \mathrm{kg}$, i.v.) em um intervalo de $10 \mathrm{~min}$, e concomitante administração de solução coloidal de hidroxietilamido 6\% (Plasmin ${ }^{\circledR}{ }^{4}, 5 \mathrm{~mL} / \mathrm{kg}$, i.v.). Frente à ausência de correção do quadro hipotensivo houve a necessidade de terapia com fármaco vasoativo, para a qual se fez uso de dopamina $5 \mathrm{mg} / \mathrm{mL}$ (Dopacris ${ }^{\circledR}{ }^{1}$, $10 \mu \mathrm{g} / \mathrm{kg} / \mathrm{min}$ ) com posterior substituição por norepinefrina $1 \mathrm{mg} / \mathrm{mL}$ (Hyponor ${ }^{5}, 1 \mu \mathrm{g} / \mathrm{kg} / \mathrm{min}$ ) sendo que esta foi mantida durante o restante do período cirúrgico. Ao término do procedimento operatório foi realizado desmame do vasoativo e a pressão arterial sistólica se manteve próxima de $120 \mathrm{mmHg}$. Para analgesia no pós-operatório imediato foi administrada metadona $10 \mathrm{mg} /$ $\mathrm{mL}$ (Mytedon® ${ }^{1}, 0,3 \mathrm{mg} / \mathrm{kg}$, i.v.), sendo a recuperação anestésica rápida e descomplicada, apesar do animal apresentar-se deprimido nas primeiras $24 \mathrm{~h}$.

A estratégia terapêutica instituída para o paciente foi o uso dos seguintes fármacos: enrofloxacina 2,5\% (Flotril ${ }^{6}, 5 \mathrm{mg} / \mathrm{kg}$, BID, i.m.) por 7 dias; cefalotina sódica $1 \mathrm{~g}$ (Ceflen ${ }^{7}, 20 \mathrm{mg} / \mathrm{kg}$, BID, i.m.) por 7 dias; metadona (Mytedon ${ }^{1}$, $0,3 \mathrm{mg} / \mathrm{kg}$, QID, i.m.) por 3 dias e meloxicam (Maxicam ${ }^{\circledR}{ }^{8}, 0,1 \mathrm{mg} / \mathrm{kg}, \mathrm{SID}$, i.m.) por 3 dias. No pós-cirúrgico e ao longo do período de recuperação foram realizados estudos ultrassonográficos (dias 0 e 1), com objetivo de detectar prováveis sangramentos, além de exames de hematologia e bioquímica sérica (Tabela 1) para acompanhamento dos parâmetros hematológicos e da função renal do paciente. Ambos os exames, no período pós-cirúrgico não demonstraram piora nos parâmetros avaliados.

\section{DISCUSSÃO}

$\mathrm{O}$ atropelamento de animais silvestres nas rodovias do país tem grande contribuição para a redução da fauna silvestre, juntamente com o desmatamento e o tráfico de animais [18].

$\mathrm{O}$ atendimento imediato e especializado a animais atropelados é de fundamental importância para a manutenção da vida do paciente, pois, os acidentes podem causar severas lesões que conduzem ao óbito de forma rápida, dessa maneira é importante o reconhecimento das prioridades de avaliação e tratamento, buscando neutralizar/controlar as alterações que impeçam as funções vitais do organismo [23]. 
Tabela 1. Parâmetros de hemograma e bioquímica sérica de exemplar de Cerdocyon thous, no início do atendimento (dia 0 ) e 13 dias após o procedimento cirúrgico.

\begin{tabular}{cccc}
\hline Variável & Dia 0 & Dia 13 & Valores de referência [11,17] \\
\hline Hemácias $\left(\right.$ milhões $\left./ \mathrm{mm}^{3}\right)$ & 3,210 & 3,110 & 4,31 a 6,77 \\
Hemoglobina $(\mathrm{g} \%)$ & 9 & 11,20 & 12,96 a 16,88 \\
Hematócrito $(\%)$ & 30 & 38 & 38 a 49 \\
VCM $^{\mathrm{a}}\left(\mu^{3}\right)$ & 93,457 & 122,186 & 68 a 95 \\
CHCM $^{\mathrm{b}}(\%)$ & 30 & 29,4 & 31 a 38 \\
Proteínas Totais $(\mathrm{g} \%)$ & 4,400 & 7,20 & 5,47 a 7,09 \\
Plaquetas $\left(\mathrm{mm}^{3}\right)$ & 20900 & 240000 & $233270 \pm 112630$ \\
Albumina $(\mathrm{g} / \mathrm{dL})$ & 1,600 & 2,90 & 2,44 a 3,98 \\
ALT $(\mathrm{UI} / \mathrm{L})$ & 540 & 71 & 12 a 52 \\
Fosfatase alcalina $(\mathrm{UI} / \mathrm{L})$ & 85 & 52,0 & - \\
Ureia sanguínea $(\mathrm{mg} / \mathrm{dL})$ & 81 & 17,0 & 22,46 a 71,84 \\
Creatinina $(\mathrm{mg} / \mathrm{dL})$ & 1,490 & 0,99 & 0,37 a 1,11 \\
\hline
\end{tabular}

aVolume corpuscular médio; 'Concentração de hemoglobina corpuscular média; 'Alanina aminotransferase.

A manutenção da patência das vias aéreas e da circulação sanguínea são as primeiras medidas a serem adotadas em animais conduzidos aos atendimentos emergenciais [23]. Para animais silvestres essas prioridades não se alteram, dessa forma, no atendimento inicial ao espécime de Cerdocyon thous, priorizou-se a avaliação das vias aéreas, e na ausência de alterações seguiu-se com a avaliação do paciente. As alterações circulatórias decorrentes de traumas, como os quadros hemorrágicos, são muitas vezes incompatíveis com a vida do animal e são consideradas as principais causas de óbito pós-traumático[19,23]. A presença dos sinais clínicos de hipotermia, hipotensão, taquicardia, mucosas de coloração pálidas e secas, e tempo de preenchimento capilar diminuído no espécime de Cerdocyon thous conduziram a suspeita do quadro de choque hemorrágico.

Nos casos de hemorragia a alteração primária é caracterizada pela diminuição do volume sanguíneo circulante e queda acentuada do retorno venoso e pressão venosa central, com posterior diminuição do débito cardíaco e quadros de hipotensão [19]. Todavia, em casos de hemorragias com quadros de hipovolemia já instalado, o organismo aciona um mecanismo compensatório de vasoconstrição periférica, que leva a diminuição de temperatura das extremidades, taquicardia e aumento da contratilidade da musculatura cardíaca, para manutenção do débito cardíaco e perfusão de órgãos prioritários [15,19].
A infusão da solução coloidal de hidroxietilamido para a correção do quadro de hipotensão e hipoalbuminenia visa aumentar o volume vascular, já que esta é uma macromolécula e pode permanecer por longos períodos na circulação sistêmica, enquanto os cristaloides são contraindicados em grandes volumes nos casos de hipovolemia por sangramento ativo [9]. A utilização de simpaticomiméticos, como a dopamina, foi necessária já que o ajuste do plano anestésico e a reposição de volume vascular não foram eficientes para correção da hipotensão $[3,13]$. A troca de vasoativo foi realizada, pois não houve melhora significativa mesmo após um longo período de infusão. O fármaco escolhido como substituto foi a norepinefrina, um fármaco com maior afinidade por receptores alfa adrenérgicos, levando a uma maior vasoconstrição periférica e aumento da pressão arterial sem prejuízo do débito cardíaco [26].

$\mathrm{Na}$ avaliação do hemograma (dia 0 ) foi possível observar queda nos valores de hemácias, hemoglobina, hematócrito e proteínas totais em relação aos valores de referência [11,17]. Além disso, foi observada discreta macrocitose e moderada hipocromia, que pode ser justificada pela necessidade do organismo em repor a volemia, ao liberar na circulação células mais jovens, que se apresentam em maior tamanho e com menor concentração de hemoglobina, que correspondem à mecanismos compensatórios da perda sanguínea $[4,20]$.

Os valores observados no exame de bioquímica sérica mostraram pequeno aumento nos valores 
de alanina aminotransferase (ALT), uréia sanguínea e creatinina. A creatinina é produto da degradação não enzimática da fosfocreatina presente na musculatura, sendo seu valor inversamente proporcional a taxa de filtração glomerular (TFG) [16]. Os valores de creatinina sérica refletem, como já citado, a TFG, porém também podem sofrer alterações por conta de lesões extensas em miócitos e quadros de desidratação [27]. No paciente atendido sugere-se que o aumento da creatinina tenha origem no quadro hemorrágico, que gerou hipovolemia e baixa perfusão renal e por consequência uma baixa na TFG, e também nas lesões musculares decorrentes do trauma. Já os níveis de ureia, quando aumentados em demasia, normalmente podem ser oriundos de disfunções na excreção urinária, problemas renais, pós-renais e quadros de desidratação [27]. Desta forma, assim como o aumento pouco significativo da creatinina, os valores encontrados para ureia podem ser justificados pelo quadro de baixa perfusão renal devido ao processo hemorrágico.

Ao considerar as complicações decorrentes desse tipo de trauma, procedimentos cirúrgicos são por diversas vezes necessários, todavia, poucos trabalhos que descrevem o emprego de técnicas cirúrgicas para animais silvestres, ou que discorram, de maneira detalhada as particularidades anatômicas destas espécies. Feranti et al. [10] abordaram o uso da técnica de nefrectomia videolaparoscópica em um espécime de graxaim do campo (Pseudalopex gymnocercus) com displasia renal, contudo não foram encontrados trabalhos que relatem o emprego da técnica de nefrectomia total unilateral para cachorro-do-mato (Cerdocyon thous), principalmente em situações emergenciais.

A técnica descrita para cães domésticos se mostrou útil e eficiente neste exemplar de Cerdocyon thous e possibilitou seu retorno à natureza 18 dias após o atropelamento, sem comprometer a função renal, conforme observado nos parâmetros de bioquímica sérica do dia 13 em relação aos de valores de referência $[11,17]$.

Cabe salientar a importância da rápida destinação destes animais a centros especializados e a execução de exames complementares que possibilitem a rápida triagem, com posterior submissão destes pacientes a intervenções terapêuticas adequadas.

\section{MANUFACTURERS}

${ }^{1}$ Laboratório Cristália. São Paulo, SP, Brazil.

${ }^{2}$ Digicare LifeWindowLW9x Vet. Rio de Janeiro, RJ, Brazil.

${ }^{3}$ Fresenius Kabi Brasil Ltda. Aquiraz, CE, Brazil.

${ }^{4}$ HalexIstar. Goiâna, GO, Brazil.

${ }^{5}$ Hypofarma. Ribeirão das Neves, MG, Brazil.

${ }^{6}$ Schering-Plough Indústria Farmacêutica. São Paulo, SP, Brazil.

${ }^{7}$ Agila Especialidades Farmacêuticas. Campos dos Goytacazes, RJ, Brazil.

${ }^{8}$ Ouro Fino Saúde Animal. Cravinhos, SP, Brazil.

Declaration of interest. The authors report no conflicts of interest. The authors alone are responsible for the content and writing of the paper.

\section{REFERENCES}

1 Becker M. \& Dalponte J.C. 2013. Rastros de Mamíferos Silvestres Brasileiros: um guia de campo. 3.ed. Rio de Janeiro: Technical Books Editora, 166 p.

2 Benn B. \& Herrero S. 2002. Grizzly bear mortality and human access in Banff and Yoho National Parks, 1971-98. Ursus. 13(1): 213-221.

3 Bistner S.I., Ford R.B. \& Raffe M.R. 2009. Manual de procedimentos veterinários e tratamento emergencial. 7.ed. São Paulo: Roca, pp.32-81.

4 Campbell T.W. 2015. Exotic Animal Hematology and Cytology. 4th edn. Ames: Wiley-Blackwell, 402p.

5 Campione F. \& Mantovani M. 1998. Estudo comparativo do uso de omento, telas de náilon e vicril, no tratamento do trauma renal em cães. Acta Cirúrgica Brasileira. 13(1): 1-4.

6 Costa S.A.B. 2014. Manejo do paciente politraumatizado na clínica de animais de companhia. 122f. Lisboa, Portugal. Dissertação (Mestrado) - Integrado em Medicina Veterinária. Universidade de Lisboa.

7 Crowe D.T. 2006. Assessment and management of the severely polytraumatized small animal patient. Journal of Veterinary Emergency and Critical Care. 16(4): 264-275.

8 Cury J., Mesquita J.L.B., Pontes J., Oliveira L.C.N., Cordeiro M. \& Coelho R.F. 2008. Trauma urológico. Revista de Medicina. 87(3): 184-194.

9 Davis H., Jensen T., Johnson A., Knowles P., Meyer R., Rucinsky R. \& Shafford H. 2013. AAHA/AAFP fluid therapy guidelines for dogs and cats. Journal of the american animal hospital association. 49(3): 149-159.

10 Feranti J.P.S., Motta A.C., Oliveira M.T., Wiecheteck F., Corrêa L.F.D., Pires J.S., Beck C.A.C. \& Brun M.V. 
2014. Nefrectomia videolaparoscópica em graxaim-do-campo (Pseudalopex gymnocercus) com displasia renal. Pesquisa Veterinária Brasileira. 34(8): 776-779.

11 Jorge R.S.P. \& Jorge M.L.S.P. 2014. Carnivora - Canidae (Cachorro-do-mato, Cachorro-vinagre, Lobo-guará e Raposa-do-campo). In: Cubas Z.S., Silva J.C.R. \& Catão-Dias J.L. (Eds). Tratado de Animais Selvagens: Medicina Veterinária. 2.ed. São Paulo: Roca, pp.764-778.

12 Júnior G.A.P., Paganelli F., Scarpelini S., Stracieri L.D.S., Féres O. \& Andrade J.I. 1998. Trauma Renal. Revista do Colégio Brasileiro de Cirurgiões. 26(1): 57-63.

13 Littaman M.P. \& Drobatz K.J. 1997. Distúrbios hipertensivos e hipotensivos. In: Ettinger S. J. \& Feldman E.C. (Eds). Tratado de Medicina Interna Veterinária. 4.ed. São Paulo: Manole, pp.134-135.

14Lucherini M. 2015. Cerdocyon thous. The IUCN Red List of Threatened Species. Fonte http://dx.doi.org/10.2305/ IUCN.UK.2015-4.RLTS.T4248A81266293.en.>[Accessed online in October 2016].

15 Marson F., Pereira Jr. G.A., Pazin Filho A. \& Basile-Filho A. 1998. A síndrome do choque circulatório. Medicina. 31(3): 369-379.

16 Martins M.F.M.R. 2014. Doença renal crónica felina: estudo sobre a receptividade dos proprietários de gatos relativamente às opções de prevenção e tratamento. 68f. Vila Real, Portugal. Dissertação (Mestrado)-Mestrado em Medicina Veterinária. Universidade de Trás-os-montes e Alto Douro.

17 Mattoso C.R.S., Catenacci L.S., Beier S.L., Lopes R.S. \& Takahira R.K. 2012. Hematologic, serum biochemistry and urinary values for captive Crab-eating Fox (Cerdocyon thous) in São Paulo state, Brazil. Pesquisa Veterinária Brasileira. 32(6): 559-566.

18 Miranda C.M.S., Nascimento A.A.T., Miranda R.F.P. \& Carvalho G.D. 2011. "Frear é o bicho: sua atenção vale uma vida!" - Importância da conscientização sobre o atropelamento de animais silvestres. V\&Z em Minas: Revista Veterinária e Zootecnia em Minas. 21(111): 20-23.

19 Nishimori C.T., Paula D.P., Moraes P.C., Conceição E.D.V., Carareto R., Nunes N. \& Freitas P.M.C. 2006. Alterações hemodinâmicas e intracranianas em cães com hemorragia aguda, anestesiados com isofluorano. Arquivo Brasileiro de Medicina Veterinária e Zootecnia. 58(6): 1048-1056.

20 Rebar A.H. 1998. Interpretación del Hemograma Canino y Felino. St. Louis: Nestlé Purina Petcare Company, 90p.

21 Reis N.R., Peracchi A.L., Pedro W.A. \& Lima I.P. 2006. Mamíferos do Brasil. Londrina: Technical Books Editora, 437 p.

22 Rocha A.C.C.L. 2008. Dieta de três espécies de carnívoros simpátricos no Parque Nacional Grande Sertão Veredas, MG e Ecologia e Comportamento do lobo-guará (Chrysocyon brachyurus, Illiger, 1815). 112f. Belo Horizonte, MG. Dissertação (Mestrado em Ciências Biológicas)-Programa de Pós-graduação Ecmvs, Universidade Federal de Minas Gerais.

23 Rohde L.M.S., Amaral B.P. \& Müller D.C.M. 2014. Caracterização de lesões e alterações clínicas de pacientes politraumatizados atendidos no hospital veterinário da Unijuí (2013-2014). In: Relatório técnico-científico da XXII Seminário de Iniciação Científica (Ijuí, Brasil). pp.1-5.

24 Rosin E. 2005. Rim: Nefrectomia. In: Bojrab M.J. (Ed). Técnicas Atuais em Cirurgia de Pequenos Animais. Cap. 24. 3. ed. São Paulo: Roca pp.346-348.

25 Sássi C.M., Nascimento A.A.T., Miranda R.F.P. \& Carvalho G.D. 2013. Levantamento de animais silvestres atropelados em trecho da rodovia BR482. Arquivo Brasileiro de Medicina Veterinária e Zootecnia. 65(6): 1883-1886.

26Silverstein D.C. \& Beer K.A.S. 2015. Controversies regarding choice of vasopressor therapy for management of septic shock in animals. Journal of veterinary emergency and critical care. (1): 48-54.

27 Stockham S.L. \& Scott M.A. 2011. Fundamentos de Patologia Clínica Veterinária. 2. ed. Rio de Janeiro: Guanabara Koogan, 729 p. 